\title{
Toward New Engagement Paradigms For Intraocular Lenses: Light-Initiated Bonding of Capsular Bag to Lens Materials
}

\author{
Susana Marcos, ${ }^{1}$ Nicolás Alejandre,,${ }^{1,2}$ Jorge Lamela, ${ }^{1}$ Carlos Dorronsoro, ${ }^{1}$ and Irene E. Kochevar ${ }^{3}$ \\ ${ }^{1}$ Instituto de Óptica, Consejo Superior de Investigaciones Científicas, Madrid, Spain \\ ${ }^{2}$ Fundación Jiménez Díaz, Madrid, Spain \\ ${ }^{3}$ Wellman Center for Photomedicine, Massachusetts General Hospital and Harvard Medical School, Boston, Massachusetts, United States
}

Correspondence: Jorge Lamela, Instituto de Óptica, Consejo Superior de Investigaciones Científicas, c/Serrano 121, 28006, Madrid, Spain; jorge.lamela82@gmail.com.

Submitted: April 10, 2015

Accepted: May 6, 2015

Citation: Marcos S, Alejandre N, Lamela J, Dorronsoro C, Kochevar IE. Toward new engagement paradigms for intraocular lenses: light-initiated bonding of capsular bag to lens materials. Invest Ophthalmol Vis Sci. 2015;56:4249-4256. DOI:10.1167/ iovs. $15-17070$
Purpose. Successful intraocular lens procedures, that is, implantation of accommodating intraocular lenses (A-IOL), require firm engagement of the IOL haptics to the capsular bag. We evaluated the use of photochemical bonding to engage IOL materials to the capsular bag.

Methods. Freshly enucleated eyes of New Zealand rabbits were used in two types of photobonding experiments using Rose Bengal (RB) photoinitiation and green light (532-nm) irradiation. First, RB-stained capsular bag strips were photobonded ex vivo to IOL polymer [poly(2-hydroxyethyl methacrylate) pHEMA] strips in an atmosphere of air and of nitrogen. Second, IOLs were implanted intracapsularly and photobonded intraocularly. Irradiation times were between 30 and 180 seconds, and laser irradiance was between 0.25 and $0.65 \mathrm{~W} / \mathrm{cm}^{2}$. The strength of the bonding was tested using a custom-developed uniaxial extensiometry system and the breakage load (the load that caused breakage per bonded area) was calculated.

REsults. The breakage load of ex vivo capsule-pHEMA bonds increased exponentially with irradiation time, using $0.45 \mathrm{~W} / \mathrm{cm}^{2}$. In air, the average breakage load across all conditions was $1 \mathrm{~g} / \mathrm{mm}^{2}$ and 1.6 times lower than that in a nitrogen atmosphere. Intraocularly, RB-stained IOLs were strongly photobonded to the capsule bag with breakage loads $>0.8 \mathrm{~g} / \mathrm{mm}^{2}$.

Concuusions. Breakage of the photobonded linkage between IOL material and capsular bag required loads substantially greater than the maximum force of ciliary muscle, suggesting that this technology may introduce a new paradigm for engagement of A-IOLs. The bonding produced in air was stronger than that in nitrogen atmosphere, suggesting that oxygen is involved in the chemical mechanism for photobonding.

Keywords: A-IOLs, lens capsule, photobonding, physiological optics

\begin{abstract}
Cataract surgery is the most common surgical procedure in ophthalmology, generally involving replacement of the crystalline lens with an intraocular lens (IOL). The standard replacement method involves implantation of the IOL inside the capsular bag following an anterior circular curvilinear capsulorhexis, which overlaps the periphery of the IOL. The stability of the IOL within the capsular bag is generally achieved by two or more plastic struts, called haptics, in the periphery of the IOL's optical zone, which produce tension in the capsular bag in the equatorial region. Although intracapsular positioning of the IOL is standard practice, extracapsular fixation is needed with damaged posterior capsule or weakened zonules. In patients with unstable posterior chamber IOLs at risk of complications such as subluxations and large IOL decentrations, IOLs may be sutured to the iris or sclera. Intraocular lens suturing remains problematic, with complications including postoperative inflammation, induced astigmatism or suture breakage, which would be minimized by a sutureless approach. Attaching IOL haptics to the sclera using plasma-derived fibrin glue $^{1,2}$ has been demonstrated, although potential drawbacks include the use of human blood products, handling difficulties of the fibrinogen-thrombin mixture, potential interactions of the thrombin in the glue with endogenous fibrinogen in the sclera, which may compromise the fibrotic healing needed to hold the lens in place after the glue degrades. ${ }^{3-5}$
\end{abstract}

Copyright 2015 The Association for Research in Vision and Ophthalmology, Inc iovs.arvojournals.org | ISSN: 1552-5783
Strong engagement of the IOL haptics to eye tissue is also required for the mechanism of action of accommodating IOLs (A-IOLs), which is intended to restore the accommodation capability of the eye. ${ }^{6}$ Accommodating IOLs use the accommodative forces transmitted from the ciliary muscle to the lens by the zonulae and the lens capsule to shift axially or laterally one or more elements or to reshape the geometry of the A-IOL. The mechanism for several A-IOL designs requires the capsular bag to mimic that in the intact eye, although it is likely that fibrosis following cataract surgery will compromise these mechanisms. A problem identified in several A-IOLs is the lack of strong connection with the capsular bag needed for adequate transfer of forces from the ciliary muscle to the action mechanism of the lens.

Some A-IOLs require that the connection between the haptics and the periphery of the capsular bag be produced by natural fibrosis occurring during the weeks following implantation. ${ }^{7}$ However, this uncontrolled process may result in a limitation of the shifting or reshaping mechanism of the AIOL. ${ }^{8,9}$ Zonular capture haptics have been proposed to favor the fusion of the capsular bag to the haptics, assisted by the natural process of fibrosis. ${ }^{10}$ This approach requires two surgical acts separated by days, in which the haptic platform and the A-IOL are implanted, respectively. 
Alternative mechanisms for engagement of the lens haptic (or lens periphery) to the edge of the capsulorhexis using mechanical blocking arms or clasps have been proposed, although a potential drawback may be tearing or rupture of the capsule. ${ }^{11}$ The use of reversible thermo-responsive bioadhesive polymers has also been proposed to engage the peripheral part of an IOL [coated with an adhesive material such as $\operatorname{poly}(N$ isopropylacrylamide) [pNIPAM] polymer] to the anterior capsulorhexis. ${ }^{12}$ The biocompatibility of pNIPAM has yet to be proven, because a nonpolymerized form, NIPAM, is toxic to neural tissue.

An unexplored alternative is the use of photochemical bonding to engage the IOL. Ideally, the bonding would involve creation of chemical bonds between the haptic plastic material and tissue, promoted by an initiating agent activated by light. Photo-activated processes are used in ophthalmology; for example, keratoconus is treated by photo-crosslinking corneal collagen, thus stiffening the cornea. ${ }^{13}$ In this procedure, formation of intrafibrillar bonds are produced by the instillation of a photosensitizer (typically riboflavin) and UV-A irradiation. ${ }^{14}$ Corneal collagen photo-crosslinking has also been demonstrated with other photosensitizers, such as Rose Bengal (RB) and green light irradiation. ${ }^{15,16}$ One advantage of the use of $\mathrm{RB}$ is that it is a US Food and Drug-approved compound widely used in ophthalmology, for example, in dry eye staining tests. ${ }^{17}$ In addition, intracapsular use of RB was shown to be nontoxic in rabbit eye models, actually reducing the rate of posterior capsular opacification. ${ }^{18}$

The use of photochemical tissue bonding using RB and green light has been demonstrated in ophthalmology for heatfree bonding repair of damaged tissue, thus replacing sutures or staples. ${ }^{19,20}$ It is thought that activation of the photoinitiator by light absorption produces structural changes in the amino acids of the proteins of the tissue and formation of covalent bonds between collagen molecules on opposing surfaces of the two tissues in contact. ${ }^{21,22}$

In this study we explored the possibility of using a photochemical process to produce bonding between ocular tissue (capsular bag) and a polymer material typically used in IOLs [poly(2-hydroxyethyl methacrylate) pHEMA]. Achieving secure fixation of lens haptics by means of a light-induced, nontoxic process is of interest in ophthalmology. In particular, strong engagement of the lens haptics to the capsular bag will undoubtedly contribute to the success of accommodating IOL concepts, currently limited by the lack of efficient transmission of ciliary muscle forces into the lens mechanisms due to improper capsular bag-IOL engagement.

\section{METHODS}

\section{Rabbit Eyes}

A total of 48 freshly enucleated eyes of New Zealand rabbits were used. All rabbits were 3 months old and weighed 2.5 to $3.5 \mathrm{~kg}$ at the time of euthanasia. Eyes were obtained from a farm associated with the Universidad Complutense School of Veterinary Medicine, Madrid, following protocols approved by the Institutional Review Boards and Association for Research in Vision and Ophthalmology Statement for the Use of Animals in Ophthalmic and Vision Research.

\section{Photobonding System: Lamp and Photoinitiator}

A light delivery system was implemented specifically for this experiment, with minor differences in design from the system described by Verter et al. ${ }^{20}$ The light source consisted of a pumped solid-state green laser source (provided by CNI Tech,
Co. Ltd., Changchun, China), with a central wavelength of 532 $\mathrm{nm}$ and an output power of $1100 \mathrm{~mW}$ at the end of the fiber. The fiber tip is placed in the focal point of a $150-\mathrm{mm}$ focal length lens. The sample holder is placed 1 focal length after the collimating lens. The laser irradiance at the sample plane was varied using neutral density filters, between 0 and $0.65 \mathrm{~W} / \mathrm{cm}^{2}$.

The photoinitiator $\mathrm{RB}$ was prepared as a $0.1 \%$ solution by dissolving $0.01 \mathrm{~g}$ commercial RB sodium salt (Sigma-Aldrich Corp., St. Louis, MO, USA) into $10 \mathrm{~mL}$ 0.01-M phosphatebuffered solution.

\section{pHEMA Polymers and IOLs}

The polymer material used was a copolymer of pHEMA and a co-acrylamide (Vistaflex Advantage +49 ; provided by Vista Optics Ltd., Gorsey Lane, Widnes, UK), which we will refer to as pHEMA. Samples of the copolymer material were cut, using a precision optics diamond-fiber cutter, into $5-\times 10-\mathrm{mm}$ rectangular strips of 1-mm thickness. The water content of the material is $49 \%$ in the fully hydrated state. Dehydrated pHEMA samples were rehydrated in a $0.1 \% \mathrm{RB}$ solution. Some of the experiments involved the use of 2-plate haptic pHEMA-MMA (poly(2-hydroxyethyl methacrylate)-methyl methacrylate) lenses (Akreos; Bausch and Lomb, Bridgewater, NJ, USA.

\section{Capsular Bag Strip-Polymer Strip Photobonding Experiments}

Sample Preparation. Capsular bags were obtained from freshly enucleated New Zealand albino rabbit eyes, less than 12 hours post mortem. A circular section of the anterior capsule of the largest possible diameter $(7-10 \mathrm{~mm})$ was removed from the eye under an ophthalmological surgical microscope, using capsular scissors, and was immersed in a buffered saline solution (BSS). Strips of capsule $(5 \times 7$ - to $10-\mathrm{mm})$ were cut and reserved for testing in BSS. Before the photobonding procedure, the capsular bag strips were stained by immersion in $0.1 \% \mathrm{RB}$ solution for 2 minutes. The capsular bag strips were placed on top of the RB-dyed pHEMA strips so that approximately half of the capsular bag strip and polymer strip overlapped. Special care was taken to ensure the absence of wrinkles or folds in the capsular bag, and a good tissuepolymer surface contact. Figure 1 illustrates the different steps of the sample preparation.

Light Irradiation. Immediately following preparation, the sample holder was placed in the light delivery system. Irradiation exposure times ranged between 30 and 180 seconds, and laser irradiance settings were between 0.25 and $0.65 \mathrm{~W} / \mathrm{cm}^{2}$. After irradiation, samples were placed in BSS for 15 minutes before extensiometry testing.

Uniaxial Extensiometry. The strength of bonding was tested using a custom-developed uniaxial extensiometry system. For the purposes of this experiment, only two arms (at $180^{\circ}$ ) of an 8 -arm stretching system that simulated the mechanical forces produced by the ciliary muscle were used. Forces in the range of 0 to $150 \mathrm{~g}$ were produced by directcurrent servo-motors (Physik Instrumente GmbH \& Co., Karlsruhe, Germany) and measured using custom-developed load sensors. The system was calibrated using a $13-\mu \mathrm{m}-$ diameter fiberglass thread, with a Young's modulus of 70 GPa. The capsular bag end and the pHEMA end of the treated samples were clamped to opposite arms of the stretching system by means of custom-developed clamps. The zero-load condition was taken as the load value measured by the sensor when the sample was fixed in the stretcher arm without motor displacement. Different loads were achieved by displacing each arm in $0.2-\mathrm{mm}$ steps. The estimated load resolution of the stretcher was $0.1 \mathrm{~g}$. Unless otherwise indicated, the stretching 


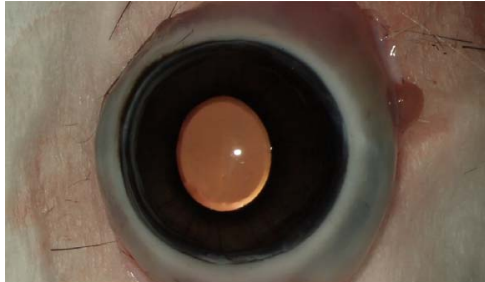

A. Remove the cornea of the eye

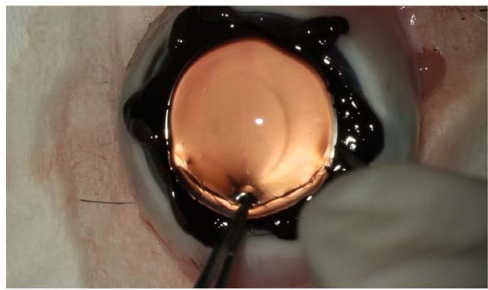

C. Cut the capsule

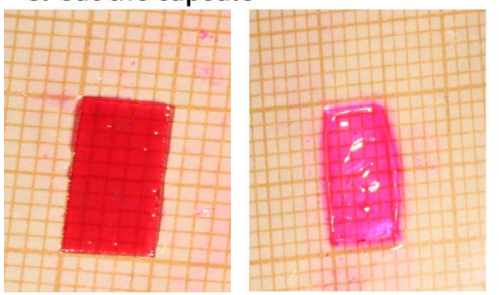

E, F. pHEMA and capsule stained with RB

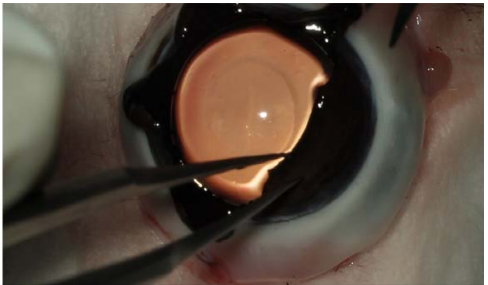

B. Remove the iris

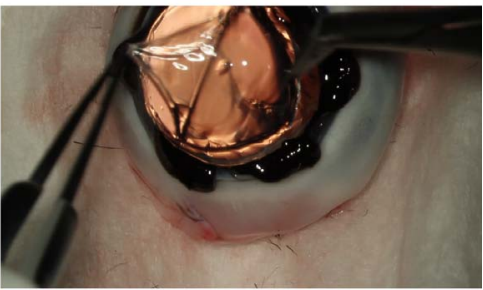

D. Remove the capsule

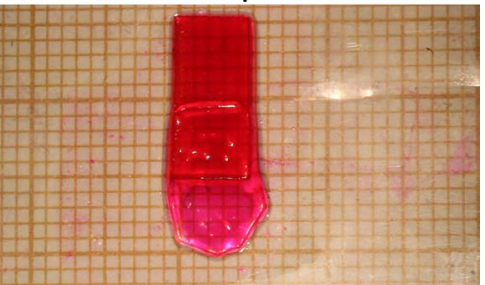

G. Final assembly

Figure 1. Sample preparation of the capsular bag and pHEMA strips for photobonding experiments. (A-D) Capsular bag removal from the rabbit's eye; (E) pHEMA stained with RB; (F) capsule stained with RB; (G) final assembly before irradiation.

was performed 15 minutes after irradiation, during which time the samples were immersed in BSS. The stretching procedure was monitored by a video camera mounted above the stretcher system and stretching was recorded for image analysis.

Capsular Tissue-pHEMA Bonding in a Nitrogen Environment. The irradiation was repeated using the same irradiance levels and exposure times in a nitrogen environment. The aim of this experiment is to test the efficiency of the procedure in the absence of oxygen. In this experiment, the capsular bag-polymer strip sample was placed in a $47 \times 22 \times$ 20-mm plastic chamber connected to a nitrogen bottle (Air Liquide España S.A., Madrid, Spain). The sample was irradiated after the air in the chamber was replaced with nitrogen through a constant flow of 0.1 bar for 5 minutes. The estimated light losses from reflection by the chamber upper wall were less than $10 \%$. The sample was placed in a humidified chamber, and sample hydration was maintained during the irradiation by introducing a BBS solution-soaked cotton in the chamber.

Data Analysis. The load required to break the capsular bag-pHEMA system was measured. The load was recorded when the capsular bag and pHEMA separated completely. In some cases, the capsular bag ruptured before the bonded area came apart, and therefore, the recorded load represents a minimum limit for breakage load. The breakage load was analyzed as a function of irradiation exposure time and fluence.

The photobonding breakage load was calculated as the ratio of load-producing breakage-to-area of bonding (i.e., area overlap between the capsular bag and pHEMA). The area of bonding was estimated from the video recordings of the sample mounted in the stretching device (in the zero-load stage) using custom-developed image analysis routines developed in ImageJ software (US National Institutes of Health, Bethesda, MD, USA). The shape of the bonded area prior to application of the load was selected by using the computer mouse, and the area was estimated using a pixel scale factor of $0.07-\mathrm{mm} /$ pixel.

Other Control Tests. Reproducibility. The series of experiments of capsular bag-pHEMA bonding in air were conducted during experimental sessions on 5 different days, and those in a nitrogen environment were conducted on 4 different days. We conducted a reproducibility test using five capsular bag samples in air, conducted in identical conditions during a single experimental session, for a fixed fluence $(0.45$ $\mathrm{W} / \mathrm{cm}^{2}$ ) and irradiation time ( 90 seconds). Reproducibility of the procedure was assessed by the standard deviation of the measurements.

Capsular Bag Tests. The influence of the procedure on capsular integrity was assessed by repeating the experiments (in air) on capsular bag strips alone $(n=3)$, either after staining with RB only or after both staining $(n=3)$ and light irradiation $\left(0.65 \mathrm{~W} / \mathrm{cm}^{2}\right.$; 90 -second exposure time; $\left.n=3\right)$. Capsular bag strips were mounted on the stretching system, and the loads required to break the treated capsular bag were measured.

Assessment of Potential Thermal Effects. The temperature attained during light irradiation was measured using a multimeter with a temperature probe (Promax Electronica S.L., Spain) with $0.5 \%$ measurement precision placed over the pHEMA strip. Measurements were performed immediately after irradiation to prevent the heating of the probe by direct incidence of laser light, for fluences ranging between 0.25 and $0.65 \mathrm{~W} / \mathrm{cm}^{2}$ and exposure times between 30 and 180 seconds. In addition, the experiment was repeated in air without application of the $\mathrm{RB}$ photoinitiator, to eliminate the occurrence of photothermal effects.

Long-Term Resistance. To assess the effectiveness of bonding over a longer period, one capsular bag-pHEMA sample $\left(0.65 \mathrm{~W} / \mathrm{cm}^{2}\right.$ fluence, 150 -second exposure time) was 

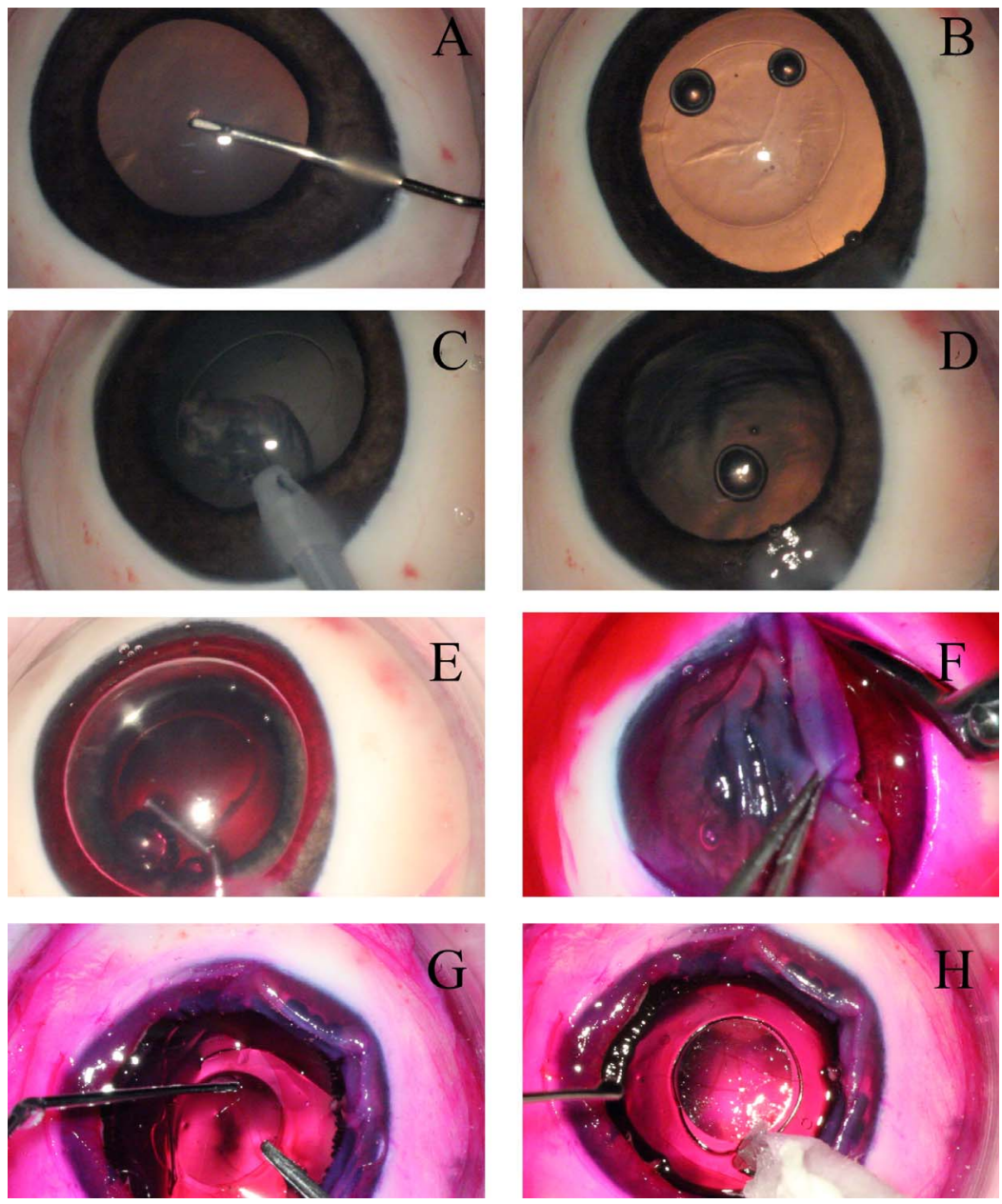

Figure 2. Preparation for intraocular bonding in a rabbit eye following corneal removal. (A, B) Capsulorhexis creation without removing the cornea, as in conventional cataract surgery; (C, D) lens material aspiration; (E) capsular bag filling with RB; (F) cornea and iris removal; (G, H) IOL implantation, centered on the capsulorhexis before irradiation, illustrating the setting immediately before irradiation.

kept in BSS solution, and the tensiometry measurements were performed at 16 days and at 7 months after the procedure.

\section{Intraocular Photobonding Experiments}

Two experiments were conducted as initial steps toward demonstration of the feasibility of the bonding of the haptics of an intraocular lens to the anterior lens capsule intraocularly.

Intraocular Bonding of the Haptics of a pHEMA Intraocular Lens to the Anterior Lens Capsule. Intact, enucleated fresh rabbit eyes ( $<4$ hours post mortem) were used. Under an operating surgical microscope, the cornea was cut, and the crystalline lens material was aspirated using a cannula through 5-mm-diameter anterior lens capsulorhexis (Simcoe). A pHEMA-MMA intraocular lens (Akreos; Bausch and Lomb) was dehydrated and rehydrated in a $0.1 \% \mathrm{RB}$ solution for 1 hour and then inserted into the capsular bag, which had been prestained with $0.1 \% \mathrm{RB}$ for 2 minutes. An air bubble (approximately $1 \mathrm{~mL}$ ) was infused in the vitreous cavity of the eye by using a 25-gauge needle. The air bubble created pressure between the anterior and posterior capsule inner wall and the IOL optic and haptics. Figure 2 illustrates the steps of the procedure. The whole eye was immersed in saline solution in a cuvette, and the cuvette was placed under the light delivery system. The cuvette was shifted laterally such that the optical axis of the instrument was $2-\mathrm{mm}$ off center from the IOL apex. The estimated peak irradiance was $0.25 \mathrm{~W} / \mathrm{cm}^{2}$ at the location of one haptic and $0.05 \mathrm{~W} / \mathrm{cm}^{2}$ at the location of the second haptic. Exposure time was 300 seconds.

In a different eye, the IOL implantation was done under similar conditions, but in this case, the capsular bag was stained with a solution of $0.1 \% \mathrm{RB}$ using a 30-gauge cannula during the hydro-dissection maneuver (injection of RB in the plane between the capsular bag and the lens cortex) after the anterior capsulorhexis was performed. The IOL was then implanted through the capsulorhexis into the capsular bag. As in the former procedure, after exposure, the IOL was cut into two pieces inside the capsular bag.

Intraocular Photobonding of the Haptics of a pHEMA Intraocular Lens to the Equator Lens Capsule Through Scleral Window Illumination. An intact, enucleated fresh rabbit eye ( $<4$ hours post mortem) was used. The cornea and iris were removed, and a scleral window was created to expose the lens equator. Then the capsular bag was emptied through a 6-mm-diameter capsulorhexis, and the capsule was stained with RB during the hydro-dissection maneuver. Then a 2-plate haptic pHEMA-MMA lens, rehydrated in RB, was introduced through the rhexis and placed against the equator with a forceps. In that position, irradiation was performed through the scleral window $\left(0.65 \mathrm{~mW} / \mathrm{cm}^{2}, 150\right.$ seconds). Then a silicone tube was glued to the pHEMA piece with cyanoacrylate. Figure 3 illustrates the different steps of the procedure. To 

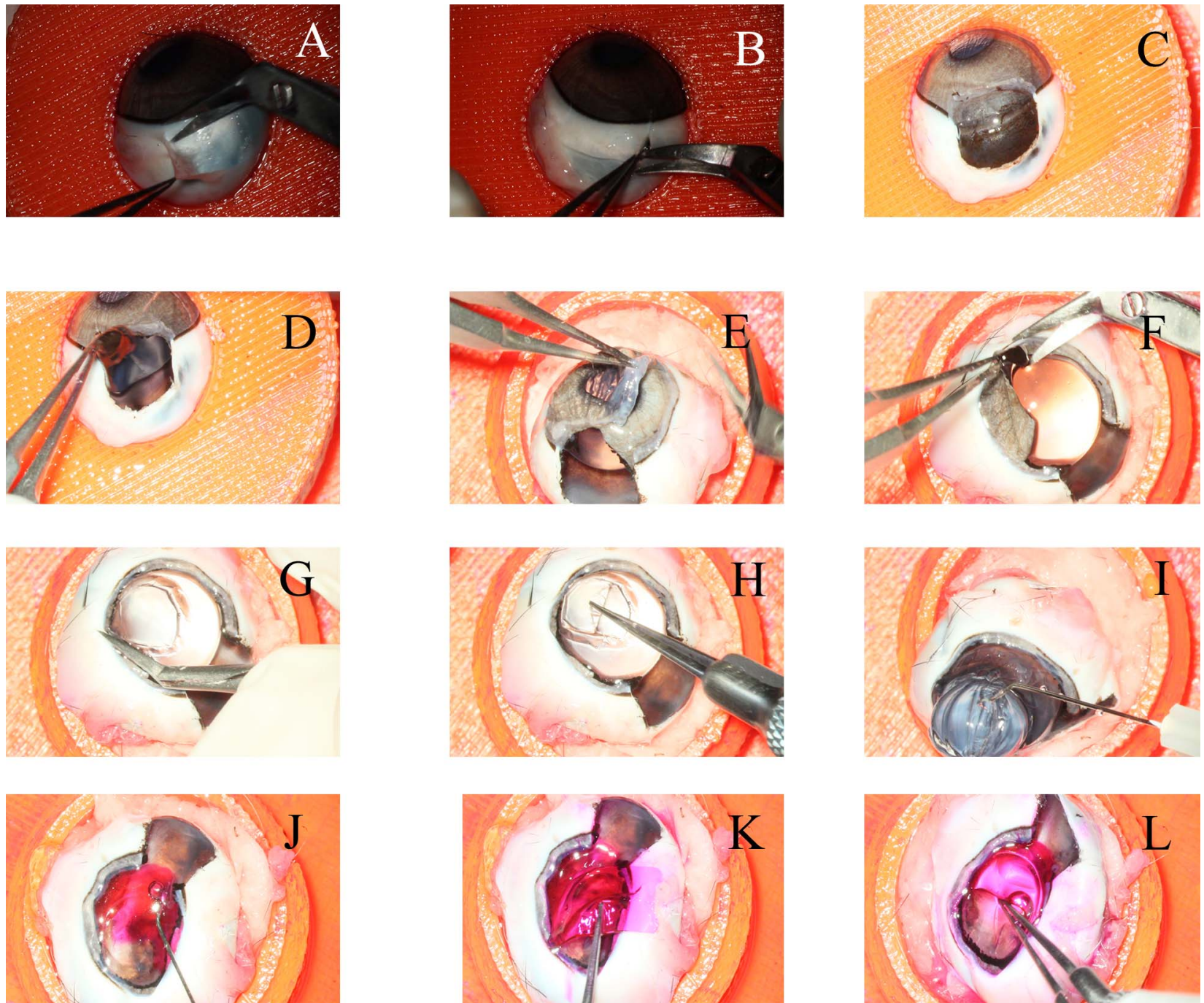

Figure 3. Eye preparation for intraocular bonding through scleral window illumination. (A-C) Removal of the conjunctiva and marking and cutting of the sclera (without cutting the choroid to avoid damaging the lens); (D-F) choroidal removal (G-I) capsulorhexis and extraction the nucleus of the lens; (J, K) capsular bag filling with RB and RB-stained implantation; (L) IOL placement against the capsular equator.

evaluate the strength of photobonding, the sclera and silicone tube were clamped to the two arms of the stretcher.

\section{RESULTS}

\section{Capsular Bag Strip-pHEMA Strip Photobonding in Air and Nitrogen}

Figure $4 \mathrm{~A}$ shows the photobond-breaking loads that caused breakage of the capsule-pHEMA bond in air as a function of irradiation time for three different laser irradiance levels: 0.25 , 0.45 , and $0.65 \mathrm{~W} / \mathrm{cm}^{2}$. Increasing exposure time and laser irradiance levels increased the breakage load. In the series of experiments illustrated in Figure 4 , the average overlapped pHEMA-capsular bag area was $16.32 \mathrm{~mm}^{2}$ (ranging from $7.55-$ $26.67 \mathrm{~mm}^{2}$ ). The average photobond-breaking load across all conditions was $1 \mathrm{~g} / \mathrm{mm}^{2}$. For a fixed fluence of $0.45 \mathrm{~W} / \mathrm{cm}^{2}$, the photobond-breakage load increased exponentially with exposure time ( $r=0.98 ; \chi^{2}$ test $\left.=\sim 1 \mathrm{E}-9\right)$ (Fig. $4 \mathrm{~B}$ ).

Figure 5 compares the photobond-breaking loads that caused breakage of the capsule-pHEMA bonding in air and in nitrogen environments. Breaking load values were normalized by the photobonded area, which was, on average, $15.41 \pm$
$4.54 \mathrm{~mm}^{2}$ in the experiments in air and $18.86 \pm 5.26 \mathrm{~mm}^{2}$ in the experiments in nitrogen. Data are for fluences ranging from 0.25 to $0.65 \mathrm{~W} / \mathrm{cm}^{2}$ (Figs. $5 \mathrm{~A}-\mathrm{C}$ ) and different exposure times. Figure 5 shaded bars indicate conditions where the capsular bag ruptured before the breakage of the capsular bag-pHEMAbonded area. Generally, photobond-breakage loads were consistently lower for irradiations in a nitrogen environment. For example, for an irradiance of $0.45 \mathrm{~W} / \mathrm{cm}^{2}$ and exposure time of 120 seconds, the photobonded area broke at a load 1.63 times higher in air than in a nitrogen environment. Furthermore, the capsule ruptured more frequently when higher irradiances were used; only three capsules ruptured at an irradiance of $0.45 \mathrm{~W} / \mathrm{cm}^{2}$ and seven at $0.65 \mathrm{~W} / \mathrm{cm}^{2}$, of a total of 33 samples.

\section{Intraocular Photobonding of Intraocular Lenses}

The IOL was successfully implanted inside the capsular bag in the procedure illustrated in Figure 2. After exposure, the IOL was cut into two pieces inside the capsular bag. Strong bonding was achieved between the anterior capsular bag and the haptic of the lens that had been exposed to the higher irradiance, whereas no significant bonding occurred for the haptic exposed 

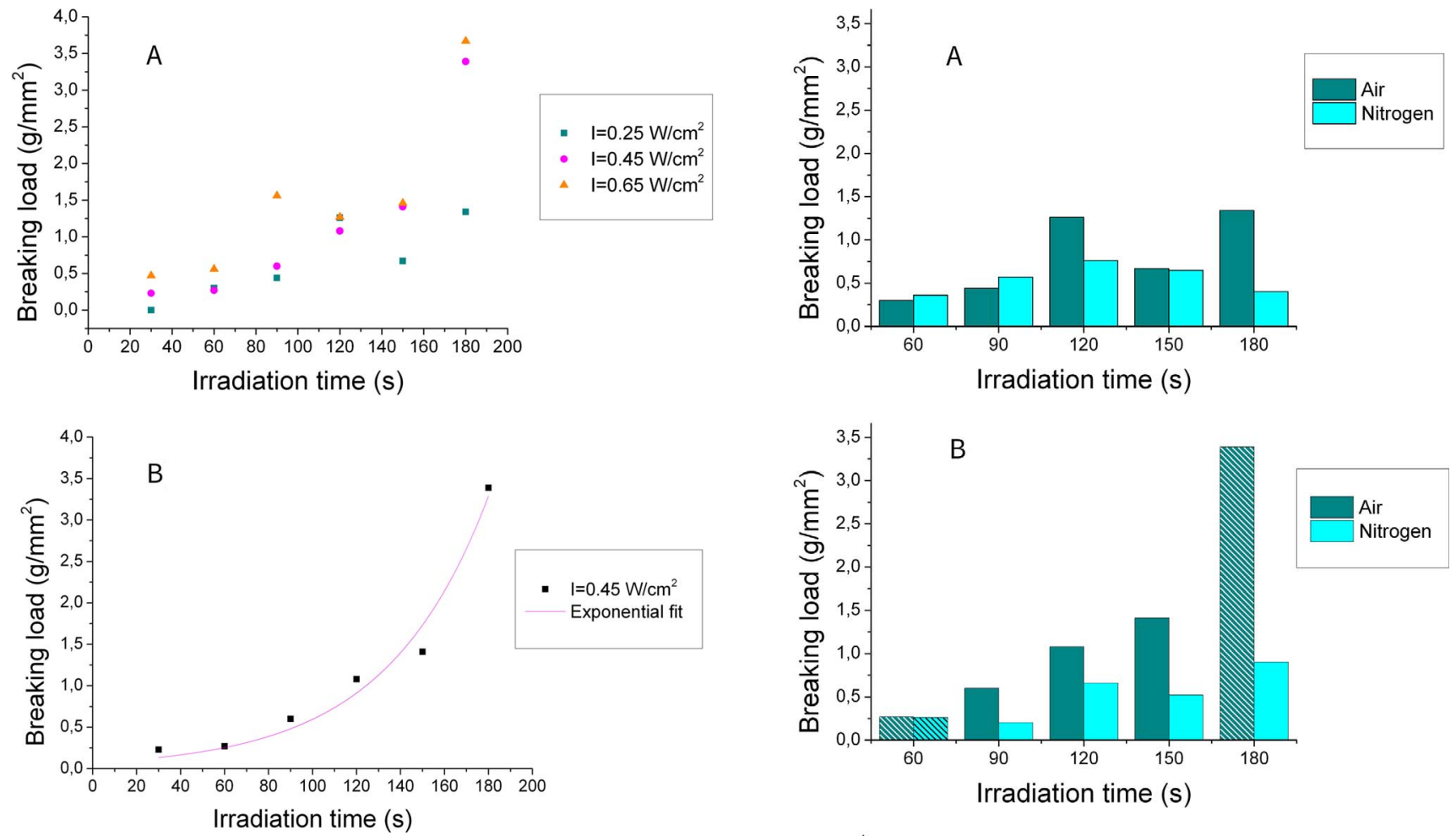

FIGURE 4. (A) Photobonding breaking load per area that produced breakage of the capsule-pHEMA bonding in air, as a function of irradiation time for three different irradiation levels $\left(0.25 \mathrm{~W} / \mathrm{cm}^{2}\right.$ [green squares]; $0.45 \mathrm{~W} / \mathrm{cm}^{2}$ [pink circles]; $0.65 \mathrm{~W} / \mathrm{cm}^{2}$ [orange triangles]. (B) Exponential fit of the photobonding breaking load as a function of exposure time for $0.45 \mathrm{~W} / \mathrm{cm}^{2}$ irradiation.

to the lower irradiance (see Supplementary Movie S1 for a complete description of the process). Similar results were obtained for both the procedure in which the IOL was stained with $\mathrm{RB}$ while the capsular bag was unstained and that in which the capsular bag had been previously filled with RB.

The pHEMA-MMA lens (attached to the silicone tube; I Akreos) was successfully implanted in the capsular bag in the procedure illustrated in Figure 3. The stretching experiment demonstrated that successful photobonding occurred between the IOL and the capsular bag (see Supplementary Movie S2 for a detailed description). The measured photobonding breakage load was $>0.8 \mathrm{~g} / \mathrm{mm}^{2}$.

\section{Control Tests}

Reproducibility. The mean and standard deviation of the photobond-breakage load per area in five consecutive samples treated under similar conditions (air experiment: $0.45 \mathrm{~W} / \mathrm{cm}^{2}$ irradiance, 90-second exposure time) were, respectively, $1.2 \mathrm{~g} /$ $\mathrm{mm}^{2}$ and $0.1 \mathrm{~g} / \mathrm{mm}^{2}$.

Capsular Bag Tests. Virgin capsular bag rupture occurred for loads $>30 \mathrm{~g}$. Treated capsular bags ruptured at lower loads, ranging from 16 to $19 \mathrm{~g}$ (capsular bag stained with $\mathrm{RB}$ ) to 22 to $26 \mathrm{~g}$ (capsular bag stained with RB and irradiated with a 0.65 $\mathrm{W} / \mathrm{cm}^{2}$ irradiance and 90 -second exposure time).

Temperature. Temperature measurements ranged from $26^{\circ} \mathrm{C}$ (room temperature) to $40^{\circ} \mathrm{C}$ for an irradiance of $0.25 \mathrm{~W} /$ $\mathrm{cm}^{2}$ and 180 seconds and from $26^{\circ} \mathrm{C}$ to $50^{\circ} \mathrm{C}$ for irradiances of 0.45 and $0.65 \mathrm{~W} / \mathrm{cm}^{2}$ and maximum exposure time. In addition, simple irradiance (without RB staining) did not produce a significant temperature increase (from $26^{\circ} \mathrm{C}-30^{\circ} \mathrm{C}$

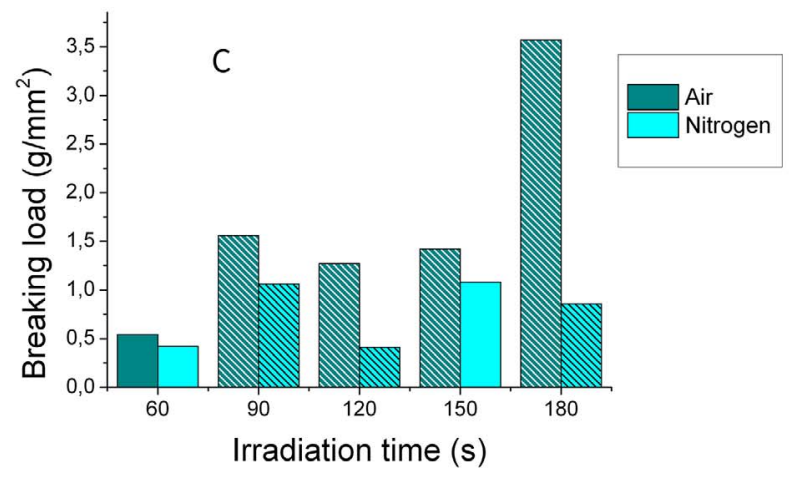

Figure 5. Comparison of the photobonding breaking load per area that produced breakage of the capsule-pHEMA bonding in air and in a nitrogen environment for three different irradiation levels, (A) $0.25 \mathrm{~W} /$ $\mathrm{cm}^{2}$, (B) $0.45 \mathrm{~W} / \mathrm{cm}^{2}$, and (C) $0.65 \mathrm{~W} / \mathrm{cm}^{2}$, as function of irradiation time. Shaded bars indicate the capsular bag ruptured before breakage of the bonded area.

under all irradiation conditions), nor did it produce measurable bonding.

Long-Term Photobonding Resistance. Bonding persisted 16 days and 7 months after treatment, with photobonding breakage loads of $3.7 \mathrm{~g} / \mathrm{mm}^{2}$ or higher.

\section{Discussion}

We have demonstrated that the haptics of an IOL can be successfully bonded to crystalline lens capsular tissue by means of light-activated photobonding processes. Loads required to rupture the bonding were more than two orders of magnitude higher than those produced internally in the eye by the ciliary muscle. ${ }^{23}$ The maximum total force capacity of the human ciliary muscle is $5 \mathrm{~g}$, and $0.05 \mathrm{~g}$ for near-vision accommodation (3-5 diopters [D]), whereas the forces applied in our experiments regardless of bonding areas ranged 
between 3 and $30 \mathrm{~g}$, obtaining that irradiation time of 60 seconds or greater, which always exceeded the maximum force of ciliary muscle. This means that exposure times greater than 90 seconds at all levels of laser irradiance tested produced a secure bonding.

At higher irradiances $\left(0.65 \mathrm{~W} / \mathrm{cm}^{2}\right)$, breakage at the overlapped photobonding site was not observed for exposure times higher than 30 seconds. Instead, for that irradiance level, the rupture occurred in the capsular bag, suggesting that treatment with RB and green light may introduce structural changes in the capsular bag, making it more brittle. A stiffer capsule may have a positive effect on the transfer of forces from the ciliary muscle to an accommodating lens mechanism. However, the implications for potential microtearing of the capsule outside the bonded should be explored, and the final laser fluence and exposure time relationships should be optimize for both bonding strength and capsular response.

Our results, therefore, suggest that this approach could be used to securely engage the haptics of an IOL to the peripheral or equatorial region of the capsular bag during accommodation. This capability is particularly interesting for accommodating IOLs, which rely on the transmission of forces from the ciliary muscle and the zonulae into the IOL equator. Also, the potential high stability of the implanted IOL fixed by photobonding may benefit IOLs, which require accurate and stable positioning, for example, for toric lenses, or in other complicated lenses requiring IOL suturing.

In principle, photobonding is potentially less toxic than other adhesive approaches proposed in the eye (e.g., use of fibrin adhesives or cyanoacrylate). ${ }^{16,24}$ The photoinitiator used is a standard of eye care used topically, ${ }^{17}$ and experimental studies in rabbit eyes have revealed that it is nontoxic intracapsularly and does not compromise corneal endothelial cell integrity. ${ }^{18}$ Exposure times and fluences of the green light used are comparable to those used in two other applications of green light and RB, namely, photobonding amniotic membrane to cornea and photo-crosslinking of the cornea. ${ }^{16,20}$ These studies have shown that exposure times and fluences did not cause retinal damage and were below the damage thresholds established by American National Standards Institute (ANSI). ${ }^{16}$ In any case, in a clinical setting, the local light exposure on the region of interest (capsular bag periphery) will be smaller than that reported in this study and will require less green light. We will, however, experimentally evaluate potential retinal damage when the treatment conditions are established.

Our results showing that strong photobonding is produced in intact lenses (rather than capsular bag strips) support the application of this technology to intraocular surgery. Settings of these proof-of-concept experiments differ substantially from those used under actual conditions, much less invasive, surgical procedures, because they required in situ illumination of the photobonding area and the bonded area was immersed in aqueous or viscoelastic materials. However, a more realistic surgical scenario could be mimicked by proper design of lens haptics and new instrumentation.

The detailed mechanism for the photobonding of capsular tissue to pHEMA remains to be established. A photochemical rather than photothermal process appears to be responsible for the bonding because only a small temperature increase $\left(<10^{\circ} \mathrm{C}\right)$ occurred, even using the highest irradiance, as previously shown for tissue-tissue photobonding. Photothermal welding of cornea requires much higher temperatures (e.g., above $50^{\circ} \mathrm{C}$ with a $\mathrm{CO}_{2}$ laser ${ }^{25}$ and $55^{\circ} \mathrm{C}-65^{\circ} \mathrm{C}$ with a 810 $\mathrm{nm}$ laser $\left.{ }^{26}\right) .{ }^{20} \mathrm{~A}$ photochemical mechanism does not produce the collagen denaturation and heat-induced peripheral tissue damage caused by photothermal bonding. The photochemical mechanism for photobonding between tissues is believed to be mediated by formation of covalent crosslinks between amino acid side chains in the collagen molecules on the surfaces of both tissues. Chitosan, a glucosamine polysaccharide, has also been photobonded to tissue by $\mathrm{RB}$ photosensitization, ${ }^{27}$ possibly by the same molecular mechanism, as chitosan contains many free amino groups. However, the chemical mechanism of photobonding of tissue and pHEMA remains to be investigated. The stronger bonding produced in the air compared to that under nitrogen atmosphere suggests that oxygen is involved in the chemical mechanism for photobonding (Fig. 5). In particular, it suggests dominance of an energy transfer from a long-lived excited RB triplet state, which would produce single oxygen and subsequent reactions of oxidized chains to form crosslinks. ${ }^{20}$ On the other hand, oxygen-independent electron-mediated reactions also may occur, as some bonding was also observed in a nitrogen environment. ${ }^{22}$

In summary, the results of our proof-of-concept study support a new paradigm for IOL engagement to the capsular bag. Appropriate haptic design, light delivery system, and surgical protocols may allow transfer of these ideas into a physically implantable device for the realization of the technique in a realistic clinical setting.

\section{Acknowledgments}

The authors thank Pablo Pérez-Merino and Daniel Pascual (Instituto de Optica, Consejo Superior de Investigaciones Científicas) for technical contributions and Hong Zhu (Wellman Center for Photomedicine) and Ulises Acuna (Instituto de Química-Física Rocasolano, CSIC) for helpful scientific discussions.

Supported by European Research Council (ERC) EU Seventh Framework Program (FP/2007-2013)/ERC grant agreement, ERC2011-AdC 294099 (SM), Spanish Government Grants FIS201125637 (SM) and FIS2013-49544-EXP (CD), and Juan de la Cierva postdoctoral fellowship (JL).

Disclosure: S. Marcos, P; N. Alejandre, P; J. Lamela, None; C. Dorronsoro, P; I.E. Kochevar, P

\section{References}

1. Agarwal A, Kumar DA, Jacob S, Baid C, Agarwal A, Srinivasan S. Fibrin glue-assisted sutureless posterior chamber intraocular lens implantation in eyes with deficient posterior capsules. $J$ Cataract Refract Surg. 2008;34:1433-1438.

2. Kumar DA, Agarwal A, Prakash D, Prakash G, Acob JS, Agarwal A. Glued intrascleral fixation of posterior chamber intraocular lens in children. Am J Opbthalmol. 2012;153:594-660.

3. Petersen B, Barkun A, Carpenter S, et al. Tissue adhesives and fibrin glues. Gastrointest Endosc. 2004;60:327-333.

4. Narang P. Modified method of haptic externalization of posterior chamber intraocular lens in fibrin glue-assisted intrascleral fixation: no-assistant technique. J Cataract Refract Surg. 2013;399:4-7.

5. Beiko G, Steinert R. Modification of externalized haptic support of glued intraocular lens technique. J Cataract Refract Surg. 2013;39:323-325.

6. Sheppard AL, Bashir A, Wolffsohn JS, Davies LN. Accommodating intraocular lenses: a review of design concepts, usage and assessment methods. Clin Exp Optom. 2010;93:441-452.

7. Glasser A. Restoration of accommodation: surgical options for correction of presbyopia. Clin Exp Optom. 2008;91:279-295.

8. Koeppl C, Findl O, Menapace R, et al. Pilocarpine-induced shift of an accommodating intraocular lens: AT-45 Crystalens. $J$ Cataract Refract Surg. 2005;31:1290-1297.

9. McLeod SD. Optical principles, biomechanics, and initial clinical performance of a dual-optic accommodating intraocular lens (an American Ophthalmological Society thesis). Trans Am Opbthalmol Soc. 2006;104:437-452. 
10. Beer PM, inventor, assignee. Accommodative intraocular lens and method of improving accommodation. US patent US2011/0307058 A1. December 15, 2011.

11. Peng Q, Yang $Y$, Zhang $X$, inventors, assignees. Accommodative intraocular lens. US Patent US-2003/0204254 A1. October 30, 2003.

12. Reisin CM, Kadziauskas KE, Basinger BC, Bumbalough TR, inventors; Abbott Medical Optics, Inc., assignee. Fixation of ophthalmic implants. US Patent US-2011/0029074 A1. February 3, 2011.

13. Spoerl E, Huhle M, Seiler T. Induction of cross-links in corneal tissue. Exp Eye Res. 1998;66:97-103.

14. Meek KM, Hayes S. Corneal cross-linking - a review. Ophthalmic Physiol Opt. 2013;33:78-93.

15. Mulroy L, Kim J, Wu I, et al. Photochemical keratodesmos for repair of lamellar corneal incisions. Invest Opbthalmol Vis Sci. 2000;41:3335-3340.

16. Cherfan D, Verter EE, Melki S, et al. Collagen cross-linking using rose bengal and green light to increase corneal stiffness. Invest Ophthalmol Vis Sci. 2013;54:3426-3433.

17. Kim J. The use of vital dyes in corneal disease. Curr Opin Opbthalmol. 2000;11:241-247.

18. Koh HJ, Kang SJ, Lim SJ, et al. The effect of photodynamic therapy with rose bengal on posterior capsule opacification in rabbit eyes. Opbthalmic Res. 2002;34:107-112.

19. Proano CE, Mulroy L, Jones E, Azar DT, Redmond RW, Kochevar IE. Photochemical keratodesmos for bonding corneal incisions. Invest Opbthalmol Vis Sci. 2004;45:21772181.

20. Verter EE, Gisel TE, Yang PG, Johnson AJ, Redmond RW, Kochevar IE. Light-initiated bonding of amniotic membrane to cornea. Invest Opbthalmol Vis Sci. 2011;52:9470-9477.

21. Shen HR, Spikes JD, Kopecekva P, Kopecek J. Photodynamic crosslinking of proteins. 1 . Model studies using histidine- and lysine-containing N-(2-hydroxypropyl) methacrylamide copolymers. J Pbotochem Pbotobiol B. 1996;34:203-210.

22. Kochevar IE, Redmond RW. Tissue repair by photochemical cross-linking. In: Hambling MR, Huang YY, eds. Handbook of Pbotomedicine. Boca Ratón, FL: Taylor \& Francis Group, LLC; 2014:771-779.

23. Fisher RF. The force of contraction of the human ciliary muscle during accommodation. J. Physiol. 1977;270:51-74.

24. Yao M, Yaroslavsky A, Henry FP, Redmond RW, Kochevar IE. Phototoxicity is not associated with photochemical tissue bonding of skin. Lasers Surg Med. 2010;42:123-131.

25. Barak A, Eyal O, Rosner M, et al. Temperature-controlled CO2 laser tissue welding of ocular tissues. Surv Ophthalmol. 1997; 42:S77-S81.

26. Matteini P, Sbrana F, Tribilli B, Pini R. Atomic force microscopy and transmission electron microscopy analyses of lowtemperature laser welding of the cornea. 2009:24:667-671.

27. Lauto A, Mawad D, Barton M, Gupta A, Piller SC, Hook J. Photochemical tissue bonding with chitosan adhesive films. Biomed Eng Online. 2010;9:47. 